Reprod. Nutr. Dévelop., 1987, 27 (1 B), 267-268.

\title{
Dégradabilité comparée dans le rumen des différentes fractions azotées des feuilles et des tiges du foin de luzerne
}

Maria R. ALVIR, J. GONZALEZ, A. ARGAMENTERIA

Departamento de Nutrición animal de la Escuela Tecnica Superior de Ingenieros Agronomos de Madrid. Universidad Politecnica de Madrid. Ciudad Universitaria, 28040 Madrid, Espagne.

Summary. The use of nylon bags showed that the kinetics of nitrogen degradation in lucerne hay stems in the rumen was different from that in the leaves due to a higher content of immediately soluble forms and of forms bound to neutral detergent fiber. The effective degradation of $\mathrm{N}$ in the stems was less than in the leaves; this difference decreased when ruminal flow rate increased.

L'objectif de ce travail est de comparer la cinétique de la dégradation ruminale de l'azote des tiges et des feuilles d'un foin de luzerne et du foin lui-même à l'aide de la technique des sachets de nylon de Mehrez et Orskov, 1977.

Matériel et méthodes. Le foin de luzerne (tabl. 1) a été récolté au cours du $2^{\circ}$ cycle de végétation et séché au sol par beau temps. Son rapport feuilles/tiges est de $82: 18$. Les sachets $(15 \times 7 \mathrm{~cm})$ ont été fabriqués en tissu de nylon $F 100$ avec double couture et soudés à la chaleur. Dans chacun on a introduit environ $3,5 \mathrm{~g}$ d'échantillon broyé à la grille de $2 \mathrm{~mm}$.

TABL. 1. - Teneur et cinétique de la dégradation de l'azote total, des parois (NDF), de la lignocellulose (ADF) et de l'azote contenu dans ces deux fractions.

\begin{tabular}{llcccccccc}
\hline & & Teneur & \multicolumn{6}{c}{ Fraction non dégradée en \% de la valeur initiale } \\
& & $(\%$ MS) & 1 & 3 & 6 & 9 & 15 & 24 & 48 \\
\hline Azote & foin & 3,07 & 57,0 & 53,3 & 44,2 & 32,7 & 19,9 & 12,1 & 10,1 \\
& feuilles & 4,44 & 61,4 & 54,8 & 45,2 & 36,8 & 24,6 & 11,4 & 5,9 \\
& tiges & 2,01 & 50,0 & 47,3 & 44,0 & 38,0 & 35,5 & 26,1 & 15,5 \\
\hline \multirow{2}{*}{ NDF } & foin & 39,71 & 93,1 & 91,6 & 84,0 & 84,3 & 72,5 & 59,0 & 57,9 \\
\hline ADF & foin & 33,07 & 90,5 & 87,5 & 80,0 & 78,2 & 69,4 & 56,4 & 55,5 \\
\hline N.NDF foin & 0,44 & 170,8 & 187,2 & 138,5 & 106,3 & 91,3 & 56,4 & 47,7 \\
\hline N. ADF foin & 0,19 & 96,1 & 128,4 & 124,7 & 110,5 & 79,4 & 62,4 & 72,3 \\
\hline
\end{tabular}

On a utilisé 4 moutons castrés, porteurs d'une fistule du rumen et nourris ad libitum avec le même foin de luzerne. Trois sachets ont été introduits au moment de la distribution du premier repas. Les sachets ont été retirés au bout de 1, 3,6, $9,15,24$ et $48 \mathrm{~h}$. Après lavage et séchage à $60^{\circ} \mathrm{C}$, on a dosé dans le résidu l'azote total, et dans le cas du foin, les parois (NDF) et la ligno-cellulose (ADF) selon la méthode de Van Soest, l'azote du NDF (N.NDF) et de l'ADF (N.ADF). 
L'ajustement des cinétiques de dégradation a été fait par rapport au modèle $y=a$ $+b e^{-c t}, y$ étant la fraction non dégradée (exprimée en \% de la valeur initiale), a, b, respectivement les fractions non dégradables et potentiellement dégradables et c la vitesse de dégradation de cette dernière.

Résultats et discussion. Les cinétiques de dégradation de l'azote total (tabl. 1) montrent des différences significatives $(P<0,05)$ entre les feuilles et les tiges pour les trois paramètres $a, b$ et $c$.

foin $y=7,666+56,992 \mathrm{e}^{-0,0938 \mathrm{t}} \quad$ ETR $=2,843$

feuilles $y=4,396+63,963 \mathrm{e}^{-0,0861 \mathrm{t}} \quad$ ETR $=3,345$

tiges $y=15,419+36,462 e^{-0,0462 t} \quad$ ETR $=3,297$.

Ces différences doivent être liées à la nature des matières azotées; ainsi l'azote des tiges se trouve en grande partie sous forme de constituants non protéiques (Fauconneau, 1960) qui sont solubles dans le liquide du rumen. Par contre, la dégradation des protéines des tiges est sensiblement plus lente que celle des feuilles parce qu'elles sont sans doute moins accessibles. Le foin se comporte de manière intermédiaire, mais beaucoup plus proche des feuilles, du fait que la plus grande partie de son azote se trouve localisé dans les feuilles.

L'évolution de la dégradabilité théorique de l'azote total en fonction de la vitesse de renouvellement $(K)$ des particules dans le rumen (Orskov et Mc Donald, 1979) est semblable pour le foin et les feuilles, avec les valeurs plus élevées que pour les tiges, la différence diminuant quand $K$ augmente. Pour une valeur de $K=0,05 \cdot h^{-1}$ la dégradabilité du foin est presque égale à celle obtenue par Cronje (1982) avec un foin de luzerne de teneur semblable en azote immédiatement dégradable mais plus élevée en azote non dégradable.

Les cinétiques de dégradation des fractions NDF et AFD du foin sont pratiquement identiques.

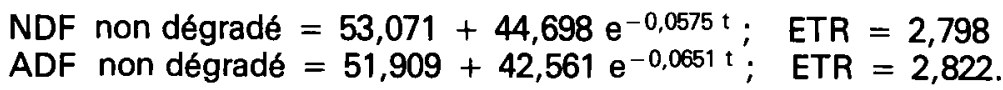

Les cinétiques de dégradation de l'azote contenu dans le NDF et l'ADF du foin sont altérées par une contamination azotée importante dans les premières heures (tabl. 1).

Cronje P.B., 1983. South. African J. anim. Sci., 13, 225-228.

Fauconneau G., 1960. Qual. Plant. Mat. végét., Vol, 3/4, 124-137.

Mehrez A. Z., Orskov E. R., 1977. J. agric. Sci, Camb., 88, 645-650.

Orskov E. R., Mc Donald I., 1979. J. agric. Sci., Camb., 92, 499-503. 